\title{
World Journal of Pediatric Surgery \\ Neonatal neuroimaging after repair of congenital diaphragmatic hernia and long-term neurodevelopmental outcome
}

Julia Kate Gunn-Charlton, ${ }^{\oplus 1,2,3}$ Alice C Burnett, ${ }^{1,3,4}$ Stephanie Malarbi, ${ }^{1,3}$
Margaret M Moran, ${ }^{1,3}$ Esther A Hutchinson, ${ }^{1,3}$ Susan Greaves, ${ }^{5}$ Rod W Hunt ${ }^{1,2,3}$

To cite: Gunn-Charlton JK, Burnett AC, Malarbi S, et al. Neonatal neuroimaging after repair of congenital diaphragmatic hernia and long-term neurodevelopmental outcome. World Jnl Ped Surgery 2019;2:e000037. doi:10.1136/ wjps-2019-000037

Received 1 January 2019 Revised 7 August 2019 Accepted 8 August 2019

Check for updates

(c) Author(s) (or their employer(s)) 2019. Re-use permitted under CC BY-NC. No commercial re-use. See rights and permissions. Published by BMJ.

${ }^{1}$ Newborn Intensive Care, The Royal Children's Hospital, Melbourne, Victoria, Australia ${ }^{2}$ Neonatal Research Group, Murdoch Children's Research Institute, Melbourne, Victoria, Australia

${ }^{3}$ Department of Paediatrics, The University of Melbourne, Melbourne, Victoria, Australia ${ }^{4}$ Victorian Infant Brain Studies (VIBeS) Group, Murdoch Children's Research Institute, Melbourne, Victoria, Australia ${ }^{5}$ Department of Occupational Therapy, The Royal Children's Hospital, Melbourne, Victoria, Australia

Correspondence to Dr Julia Kate Gunn-Charlton; julia.gunn@rch.org.au

\section{ABSTRACT}

Objective Previous outcome reports of congenital diaphragmatic hernia (CDH) have described neuroimaging anomalies and neurodevelopmental impairment. However, the link between imaging and outcome has not been described. We aimed to determine whether routine postoperative neonatal neuroimaging in infants with $\mathrm{CDH}$ detects later neurodevelopmental impairment. Methods In a prospective cohort study within a clinical service in The Royal Children's Hospital Newborn Intensive Care. Cerebral ultrasound was performed in 81 children and MRI in 57 children who subsequently underwent neurodevelopmental follow-up after surgery for CDH. MRI scans were analyzed using a scoring system designed to identify injury, maturation and volume loss. Neurodevelopmental assessment occurred at 2 years (48) and neurocognitive assessment at 5 years (26) and/or 8 years (27). Brain imaging scores corrected for gestational age at scan time were correlated with outcome measures, adjusting for known clinical confounders.

Results Clinically significant findings were identified on MRI of $16(28 \%)$ infants. Mean scores were in the normal range for all domains assessed at each age. Language impairment was seen in $23 \%$ at 2 years and verbal intellectual impairment in $25 \%$ at 8 years. Mean cognitive scores were lower in 2-year-old children with white matter injury on MRI $(p=0.03)$. Mean motor scores were lower in 2-year-old children with brain immaturity $(p=0.01)$. Associations between MRI and 5-year and 8-year assessments were no longer significant when adjusting for known clinical confounders.

Conclusions Neuroimaging abnormalities were associated with worse neurodevelopment at 2 years, but not with later neurocognitive outcomes, after accounting for clinical risk factors.

\section{INTRODUCTION}

The research focus on newborns with congenital diaphragmatic hernia $(\mathrm{CDH})$ is shifting from mortality to morbidity ${ }^{1}$ with many survivors experiencing pulmonary sequelae, gastrointestinal disorders, skeletal complications and neurodevelopmental impairment. ${ }^{2}$ Historically, reports have revealed poor outcomes, with specific clinical risk factors identified, predominantly the use of extracorporeal membrane oxygenation (ECMO), prolonged hospital length of stay (LOS), and need for oxygen and ventilation. ${ }^{3-6} \mathrm{CDH}$ is the most commonly studied single group of surgical non-cardiac patients, but reports are still based on small numbers. ${ }^{7}$ Summary data of infants with $\mathrm{CDH}$ showed that mean Bayley Scales of Infant Development second edition (BSID-II) mental and psychomotor development indices of 128 patients were one SD below the normative means. ECMO was a strong outcome predictor of worse neurodevelopment, with CT brain anomalies on $75 \%$ of patients who had received ECMO for $\mathrm{CDH}$ and neurodevelopmental delay in $42 \%$. While the 'need' for ECMO is obviously a marker of disease severity, thresholds for use of ECMO vary between centres, making it difficult to ascertain the specific ECMO impact on brain injury without multicentre studies.

More recent outcome studies describing children treated without ECMO (the norm in many institutions) are reassuring but rates of early developmental delay vary markedly depending on the assessment tools used, ${ }^{9-11}$ whereas in preschoolers reported rates of delay are consistently 20\%-35\%. ${ }^{12-15}$ Data on outcomes in school-age children report cognitive outcomes typically in the normal range, but with increased rates of attentional deficits, motor delays and decreased quality of life ${ }^{16-18}$ and high rates of academic difficulties in children aged $10-16$ years. ${ }^{19}$

Inclusive and extensive follow-up of children following $\mathrm{CDH}$ repair is recommended ${ }^{20}{ }^{21}$ but limited early intervention (EI) resources are driving the need for identification of the children at highest risk. While medical factors may be useful surrogate markers of disease severity, neonatal neuroimaging holds promise as a biomarker of brain injury at a time when clinical detection of neurological complications is challenging. However, the literature examining neonatal neuroimaging in $\mathrm{CDH}$, particularly without 


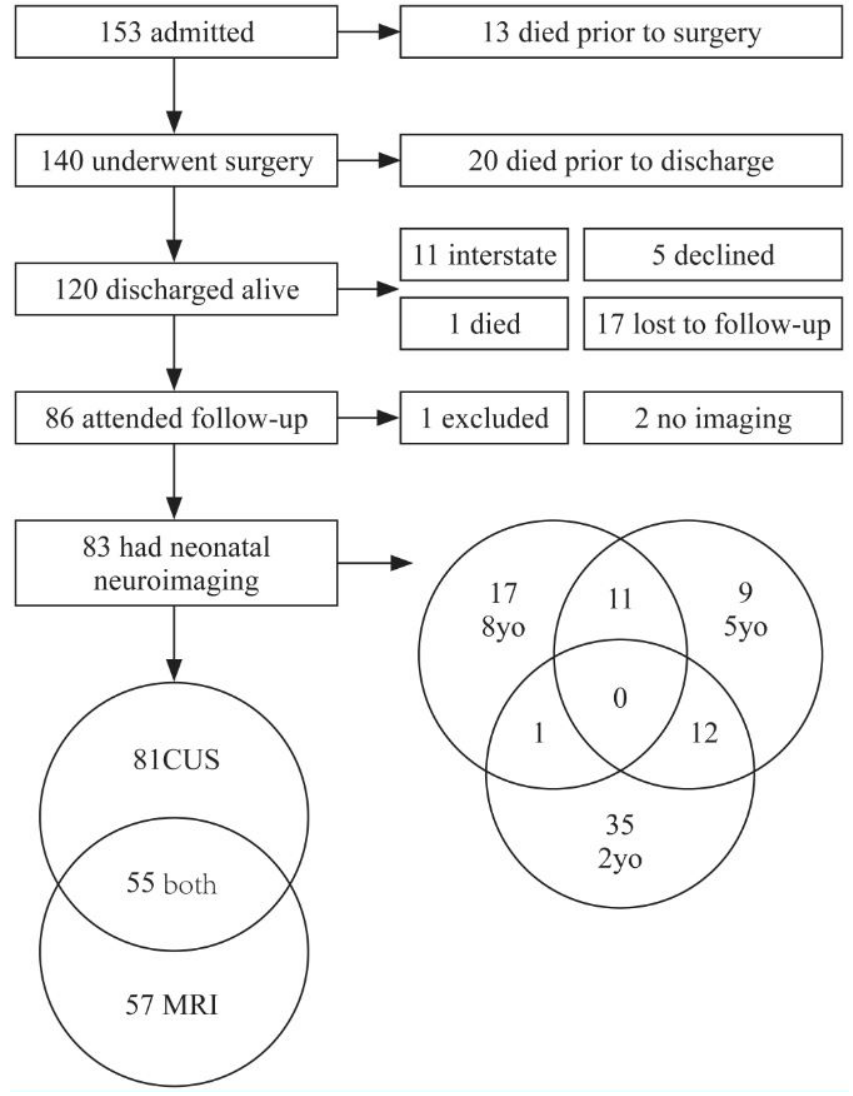

Figure 1 Included participants $(n=83)$ with overlap of imaging modalities and follow-up time frame in Venn diagrams.

the influence of ECMO, is limited with small numbers undergoing $\mathrm{MRI}^{22}$ and a lack of correlation with subsequent outcome. ${ }^{23}$ Tracy et al reported the postoperative neuroimaging findings in 42 infants with $\mathrm{CDH}$. All had cranial ultrasound, with $33 \%$ demonstrating anomalies; seven underwent MRI and all had reported abnormalities and 14 underwent CT with 11 revealing abnormal findings. An association between neuroimaging anomalies and 1 year neuromotor function did not persist to 3 years. ${ }^{24}$ MRI is now considered the gold standard for neonatal neuroimaging but, to our knowledge, there are no published reports of routinely performed MRI findings in relation to longer term outcomes in children with $\mathrm{CDH}$. Therefore, this study aimed to determine the relationship between routinely performed neonatal neuroimaging findings (cerebral ultrasound (CUS) and MRI) and neurodevelopmental outcomes at 2, 5 and 8 years of age in a clinical follow-up programme.

\section{METHODS}

\section{Participants}

Participants comprised surviving infants admitted to the Newborn Intensive Care Unit (NICU) at The Royal Children's Hospital, Melbourne, for management of $\mathrm{CDH}$ between 2003 and 2014 and who had both neonatal neuroimaging and long-term neurodevelopmental assessment (figure 1). Of the 140 who underwent surgery, 20
(15\%) died prior to discharge. Two children died after discharge; one prior to and one after 2-year assessment. Parents or carers were unable to be contacted for 17 children who were deemed lost to follow-up. Follow-up assessment at 2 years of age or older was performed in 86 children $(80 \%$ of eligible participants once interstate children and deaths were excluded). One child (with a normal CUS and MRI) underwent a modified neurodevelopmental assessment at age 4 due to deafness and two children had not undergone any neuroimaging. A total of 83 children were included in the following analyses.

\section{Measures}

Clinical data from the initial hospital admission were obtained from a database collated at the time of hospital discharge and included birth weight (BW), gestational age (GA) at birth, gender, laterality of $\mathrm{CDH}$ (left or right), need for a patch repair, use of ECMO, time on supplemental oxygen, time with ventilation via an endotracheal tube and hospital LOS. Surviving children were categorized as low, intermediate or high risk (of mortality) according to the $\mathrm{CDH}$ Study Group criteria. $^{25}$

CUS was routinely performed preoperatively and categorized as abnormal if a clinically significant abnormality was identified on clinical report. Cerebral MRIs were routinely undertaken once infants were medically stable postoperatively, with sedation avoided unless clinically indicated. They were performed on either a $1.5 \mathrm{~T}$ or 3T scanner depending on scanner availability and, at a minimum, included diffusion-weighted imaging, conventional $\mathrm{T} 1$ (1 mm slice thickness) and T2 $(2.5 \mathrm{~mm}$ slice thickness) sequences in the axial and coronal planes. Scoring of MRI scans was undertaken in retrospect by two neonatologists with expertise in MRI interpretation who were blinded to the initial clinical report. MRIs were scored for factors deemed to be clinically relevant based on a scoring system previously used to assess brain development in congenital heart disease. ${ }^{26}$ Signal changes related to injury detected in white matter (WM) or cortical or deep nuclear grey matter or parenchymal haemorrhage were determined on diffusion-weighted imaging, apparent diffusion coefficient map and susceptibility-weighted imaging as well as conventional T1 and T2 axial and coronal sequences. Axial and coronal sequences were used to assess myelination (scored 1-7 on T1) and cortical folding (scored 1-5 on T2). A higher score represents more advanced maturation. Ventricular dilatation and subarachnoid spaces were scored 1-4 on T2 axial and coronal sequences, respectively, and a binary subjective assessment of WM volume loss was determined on both T2 sequences. A higher score represents increased (more abnormal) cerebrospinal fluid (CSF) volume. A score of 0-3 was assigned for parenchymal injury, where 0 is no injury and 3 is extensive injury. Other sequences were not viewed due to the variability in sequences obtained, differences between scanners and the potential for findings on additional sequences to influence assessors when not all additional sequences 
were available for all patients. For patients who had more than one MRI performed, only the first postoperative scan after term-corrected GA was used.

Neurodevelopmental assessment involved the Bayley Scales of Infant and Toddler Development third Edition (Bayley-III) at age 2years undertaken by a qualified assessor. ${ }^{27}$ Normative data are available, although there are concerns that the expected mean \pm SD of $100 \pm 15$ underestimate impairment based on control groups from other studies. ${ }^{28-30}$ Intellectual functioning was assessed by neuropsychologists at 5 years with the Wechsler Preschool and Primary Scale of Intelligence, third edition, Australian standardization (WPPSI-III) ${ }^{31}$ and at 8 years using the Wechsler Intelligence Test for Children, fourth edition, Australian standardization (WISC-IV). ${ }^{32}$ Variables of interest were full scale intelligence quotient (FSIQ) and associated composite scores, which include WPPSI-III Verbal IQ (VIQ), Performance IQ (PIQ), Processing Speed Quotient (PSQ) scores, and WISC-IV Verbal Comprehension Index (VCI), Perceptual Reasoning Index (PRI), Processing Speed Index (PSI), and Working Memory Index scores. All outcome variables have an expected mean \pm SD of $100 \pm 15$, with $15 \%$ of children expected to score lower than $1 \mathrm{SD}$ below the mean $(<85)$, and $2.5 \%$ to score lower than two SD below the mean $(<70)$. All children routinely received formal audiology assessment in the first year of life and these results were documented at follow-up.

\section{Analysis}

MRI variables were dichotomized around the median score for each category: myelination (appropriate or delayed); cortical folding (appropriate or delayed); ventricular dilatation (absent or present); subarachnoid space volume (normal or enlarged); WM volume (normal or reduced) and parenchymal injury (absent or present).

Outcome measures included mean scores in each domain assessed and the proportion of children with scores 1 and 2 SDs below the expected mean from test norms in each domain. Descriptive data are reported as mean $\pm \mathrm{SD}$ and median (IQR) for parametric and non-parametric data, respectively. Associations of the previously mentioned clinical variables, as well as CUS abnormality and MRI variables, with linear and categorical measures of neurodevelopment at 2, 5 and 8 years of age were examined using $\chi^{2}$, unpaired t-tests and linear regression (where data were normally distributed) for univariate analysis. Logistic regression was used for the
Table 2 Background characteristics of perinatal period, surgery and length of stay for children who had both neuroimaging and neurodevelopmental follow-up.

\begin{tabular}{|c|c|}
\hline Demographic & $\begin{array}{l}\text { Included } \\
\text { patients }(n=83)\end{array}$ \\
\hline Male gender $n(\%)$ & $49(59 \%)$ \\
\hline Left-sided hernia n (\%) & $70(84 \%)$ \\
\hline Mean gestational age at birth $\pm \mathrm{SD}$ (weeks) & $38.0 \pm 2.5$ \\
\hline Mean birth weight $\pm S D(g)$ & $3009 \pm 677$ \\
\hline Median age of surgery (days) & 4 (IQR 2-6) \\
\hline Patch repair n (\%) & $18(22 \%)$ \\
\hline $\begin{array}{l}\text { Extracorporeal membrane oxygenation } n \\
(\%)\end{array}$ & $1(1 \%)$ \\
\hline $\begin{array}{l}\text { Median ventilation via endotracheal tube } \\
\text { (days) }\end{array}$ & 10 (IQR 6-19) \\
\hline Median supplemental oxygen time (days) & 13 (IQR 4-27) \\
\hline Median hospital length of stay (days) & 29 (IQR 20-43) \\
\hline \multicolumn{2}{|l|}{ Risk of death category* } \\
\hline Low risk (<10\%) & $27(33 \%)$ \\
\hline Intermediate risk (25\%) & $33(40 \%)$ \\
\hline High risk (50\%) & $23(28 \%)$ \\
\hline
\end{tabular}

${ }^{*}$ Risk of death score based on combination of birth weight $<1500 \mathrm{~g}, 5 \mathrm{~min}$ Apgar $<7$, severe pulmonary hypertension on echo, major cardiac anomaly, chromosomal anomaly. ${ }^{25}$

multivariable analysis; full-term was defined as exactly 40 weeks' gestation and corrected GA at the time of MRI scan (in days) was used as a covariate for all multivariable analysis. To determine whether the imaging findings had unique predictive value beyond known clinical risk factors, each medical variable identified on univariate analysis as associated with later outcomes $(\mathrm{p}<0.05)$ was included in the respective multivariable model.

\section{RESULTS}

Of the 83 children included in this analysis, neurodevelopmental assessments were performed at 2 years in 48 children, and cognitive assessments at 5 years in 32 children and 8 years in 29 children. Eleven children aged 8 years had previous 5-year-old assessments; one (aged 8 years) and 12 (aged 5 years) children had undergone 2-year-old assessments (figure 1). The table 1 shows the distribution of CUS and MRI with subsequent

\begin{tabular}{|c|c|c|c|c|}
\hline Imaging modality & $\begin{array}{l}2 \text {-year-old } \\
\text { children }(n=48)\end{array}$ & $\begin{array}{l}5 \text {-year-old } \\
\text { children }(n=32)\end{array}$ & $\begin{array}{l}\text { 8-year-old } \\
\text { children }(n=29)\end{array}$ & Total children/total scans \\
\hline Ultrasound & $47 / 78$ & $32 / 40$ & $28 / 34$ & $81 / 119$ \\
\hline MRI & $31 / 35$ & $21 / 21$ & $21 / 23$ & $57 / 65$ \\
\hline Total children/scan numbers & $48 / 113$ & $32 / 61$ & $29 / 56$ & $83 / 182$ \\
\hline
\end{tabular}




\begin{tabular}{|c|c|c|}
\hline & Preoperative & Postoperative \\
\hline $\begin{array}{l}\text { Patients with } \\
\text { ultrasound (81) }\end{array}$ & $\begin{array}{l}68 \text { normal } \\
1 \text { non-significant } \\
\text { abnormality } \\
0 \text { abnormal }\end{array}$ & $\begin{array}{l}6 \text { normal } \\
11 \text { non-significant } \\
\text { abnormality } \\
10 \text { abnormal }\end{array}$ \\
\hline $\begin{array}{l}\text { Patients with } \\
\text { MRI (57) }\end{array}$ & $\begin{array}{l}7 \text { normal } \\
0 \text { non-significant } \\
\text { abnormality } \\
0 \text { abnormal }\end{array}$ & $\begin{array}{l}36 \text { normal } \\
5 \text { non-significant } \\
\text { abnormality } \\
16 \text { abnormal }\end{array}$ \\
\hline
\end{tabular}

neurodevelopmental assessment at 2 years or cognitive assessment at 5 or 8 years.

The table 2 shows the demographics of the cohort. There were no differences between any of these parameters and those of the total cohort of 120 children who survived to discharge.

Summary of imaging findings is shown in table 3.

\section{Cerebral ultrasound}

Perioperative CUS was performed in 81 children (range 1-6 scans per child). The median age of the first scan was 1 day (IQR 1-2), 69 of which were preoperative. Only 15 infants who had preoperative CUS had postoperative CUS such that postoperative scans were not likely to be representative of findings from the whole cohort. Eleven (41\%) patients with postoperative CUS had findings considered not to be of clinical significance, including grade I intraventricular haemorrhage (IVH), lenticulostriate vasculopathy, slit-like ventricles and unilateral lateral ventricle prominence. Ten $(12 \%)$ had findings considered to be of clinical significance: focal WM echogenicity $(n=6)$; focal grey matter echogenicity $(n=2)$; dilated ventricles/prominent extra-axial spaces $(n=2)$. However, only five of these infants also had an MRI with which to correlate findings; the latter two of which were abnormal.

\section{Magnetic resonance imaging}

MRI was undertaken in 57 children ( 65 scans) at a median age of 31 (IQR 22-51) days. This equated to a median of 22 post-term equivalent days (IQR $6-44)$. Five (9\%) had findings on postoperative MRI considered not to be of clinical significance, including grade I IVH, mild extraaxial haemorrhage consistent with that found in healthy newborn infants and a choroid plexus cyst. Sixteen (28\%) had findings considered to be of clinical significance:

- Focal T2 hyperintensities in deep WM $(\mathrm{n}=7)$, one of which was extensive; all had normal ultrasound scans.

- Prominent CSF spaces $(\mathrm{n}=8)$; two were detected on ultrasound.

The median myelination score was 6 (IQR 3-6) and median cortical folding score was 2 (IQR 2-4) (figure 2). Nine $(16 \%)$ children had an injury score of $1(n=7)$ or 2 $(n=2)$; none had a score of 3 .
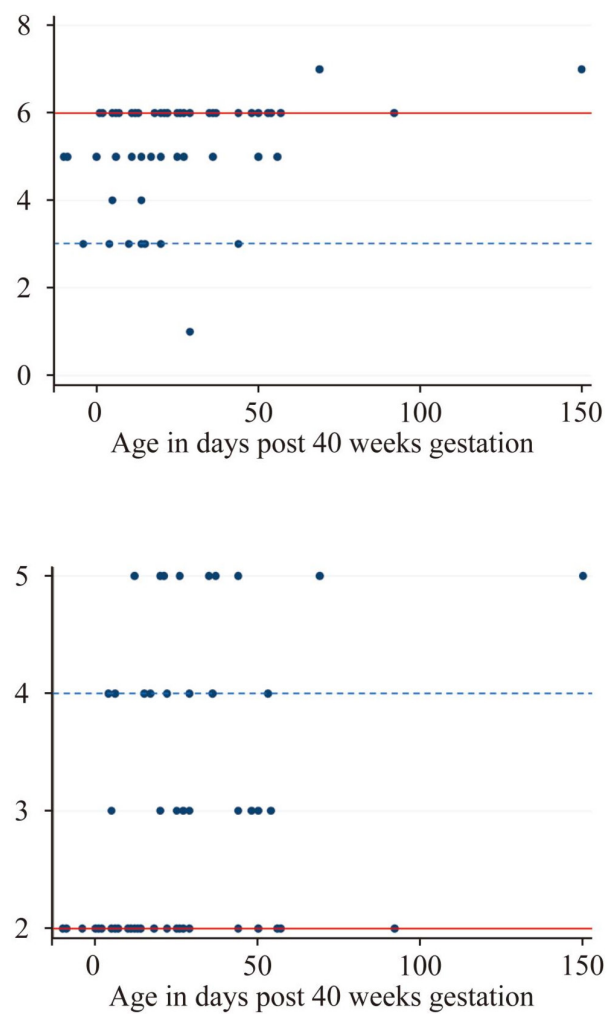

Figure 2 Myelination score and cortical folding scores relative to post-term equivalent age in days. Red line is median score and dashed blue line is IQR.

\section{Summary of neurodevelopment findings}

The table 4 shows summary scores for each age group. Mean working memory index scores at 8 years were in the normal range $(97 \pm 14)$ with no associations found between imaging and working memory findings. Eight $(10 \%)$ of children had a degree of hearing impairment on formal testing.

Relationship of neonatal imaging and medical variables with neurodevelopment in each age group

Two-year-old children

On univariate analysis, patient risk factors associated with a reduced 2-year-old score in all domains $(\mathrm{p}<0.05)$ were male gender, lower BW, need for a patch repair, ventilation time, time on supplemental oxygen and longer LOS. Lower GA at birth was associated with lower mean language and motor scores. On all outcome measures, risk factors for a score $<85(\mathrm{p}<0.05)$ were lower $\mathrm{BW}$, longer ventilation time, longer time on oxygen and longer LOS. Lower GA at birth was also a risk factor for a language or motor score $<85$. Substitution of GA as an interdependent variable for BW did not change the subsequent findings.

An ultrasound with clinically significant abnormalities was associated with lower scores in all domains and an increased risk of a cognitive or motor score $<85(\mathrm{p}<0.05)$.

Mean cognitive scores were 26.1 (95\% CI 2.4 to 49.7 ) points lower in 2-year-old children with WM injury on MRI $(p=0.03)$. This remained significant when accounting 
Table 4 Neurodevelopmental findings in children aged 2 years, 5 years and 8 years

\begin{tabular}{lllll}
\hline 2-year-old children $(\mathbf{n}=\mathbf{4 8})$ & Cognitive DQ & Language DQ & Motor DQ & \\
\hline Mean score and SD & $100.3 \pm 20.5$ & $98.9 \pm 22.6$ & $96.6 \pm 17.3$ & \\
\hline $\mathrm{N}(\%)<85$ & $8(17 \%)$ & $11(23 \%)$ & $9(19 \%)$ & \\
\hline $\mathrm{N}(\%)<70$ & $3(6 \%)$ & $7(15 \%)$ & $4(8 \%)$ & PSQ \\
\hline 5-year-old children $(\mathbf{n}=\mathbf{2 6})$ & FSIQ & VIQ & PIQ & $107.4 \pm 14.3$ \\
\hline Mean score and SD & $102.9 \pm 12.3$ & $101.2 \pm 12.1$ & $104.3 \pm 14.2$ & $1(4 \%)$ \\
\hline N (\%) $<85$ & $2(7 \%)$ & $1(4 \%)$ & $4(15 \%)$ & $0(0 \%)$ \\
\hline N (\%) $<70$ & $0(0 \%)$ & $1(4 \%)$ & $0(0 \%)$ & PSI \\
\hline 8-year-old children $(\mathbf{n}=27)$ & FSIQ & VCI & PRI & $99.8 \pm 17.4$ \\
\hline Mean score and SD & $99.7 \pm 19.2$ & $96.4 \pm 21.6$ & $101.4 \pm 17.8$ & $5(18 \%)$ \\
\hline$N(\%)<85$ & $4(15 \%)$ & $7(25 \%)$ & $3(11 \%)$ & $1(4 \%)$ \\
\hline$N(\%)<70$ & $2(7 \%)$ & $3(11 \%)$ & $1(4 \%)$ & \\
\hline
\end{tabular}

DQ, developmental quotient; FSIQ, full-scale IQ; PIQ, performance IQ; PRI, perceptual reasoning index; PSI, processing speed index; PSQ, processing speed quotient; $\mathrm{VCI}$, verbal comprehension index; VIQ, verbal IQ.

for BW, gender, oxygenation time and LOS. An association was seen between increased ventricular dilatation and poorer mean cognitive scores (coefficient -20.7 (95\% CI -28.1 to 3.3$) ; \mathrm{p}=0.02$ ), but this was no longer significant once adjustment was made for BW, gender, time on supplemental oxygen and LOS. Similarly, an association was found between degree of cortical folding and mean language scores (coefficient 18.1 (95\% CI 0.4 to 35.9$) ; \mathrm{p}=0.04$ ) but this was no longer significant once adjustment was made for the other factors previously mentioned. Mean motor scores were 24.6 (95\% CI 5.4 to $43.8)$ points lower in 2-year-old children who had myelination delay $(\mathrm{p}=0.01)$ and $18.7(95 \% \mathrm{CI} 5.9$ to 31.4$)$ points lower in those with cortical folding delay $(\mathrm{p}=0.01)$ and these remained significant once adjustment was made for clinical factors.

Two-year-old children with a motor score $<85$ were more likely to have myelination delay $\left(\chi^{2}=7.6, \mathrm{p}=0.01\right)$ and delay in cortical folding $\left(\chi^{2}=5.4, p=0.02\right)$. Only the cortical folding remained significantly associated once accounting for other factors. In fact, all nine children with a motor score $<85$ had cortical folding delay.

\section{Five-year-old children}

On univariate analysis, risk factors identified for lower scores $(p<0.05)$ were need for a patch repair and longer ventilation time (VIQ) and male gender (FSIQ and PSQ). Risk factors for a 5 -year-old score of $<85$ were need for a patch repair (VIQ) and ventilation time (FSIQ). Neither CUS abnormalities nor any MRI parameters were associated with abnormal cognitive findings. An association was seen between cortical folding and VIQ (coefficient 15.2 (95\% CI 2.2 to 28.3 ), $\mathrm{p}=0.03$ ), but this was no longer significant when accounting for gender and ventilation time.

\section{Eight-year-old children}

On univariate analysis, patient risk factors associated with reduced 8-year-old scores in all domains $(\mathrm{p}<0.05)$ were lower GA at birth, time on oxygen and longer LOS. Additional risk factors were lower BW (FSIQ, VCI, PSI), need for a patch repair (FSIQ, PSI) and longer ventilation time (FSIQ, PRI, PSI). Risk factors for an 8-year-old score of $<85(\mathrm{p}<0.05)$ were lower GA at birth (FSIQ, PSI), lower BW (PSI), need for a patch repair (FSIQ, PSI) and longer ventilation time, time on oxygen and LOS (FSIQ, VCI, PRI, PSI). There was no association between CUS findings and 8-year outcome. More advanced cortical folding on MRI was associated with a mean VCI 20.7 (95\% CI 1.7 to 39.6) points higher $(\mathrm{p}=0.03)$, but was no longer significant when controlling for GA at birth, use of a patch repair, LOS and time on oxygen. There was no association between 8-year-old scores and other MRI variables.

\section{DISCUSSION}

We aimed to determine whether routine preoperative CUS and postoperative cerebral MRI following $\mathrm{CDH}$ repair could identify the children at highest risk of subsequent neurodevelopmental impairment. The pattern of maturation delay was consistent with that described in Danzer's cohort using an earlier version of the same tool. ${ }^{23}$ However, our rates of abnormalities identified are lower than that described by Tracy et al, despite a higher rate of MRI versus CUS use, which may relate to differences in ECMO utilization. ${ }^{24}$

Mean scores were in the normal range at each of the ages assessed. About one in four children aged 2 and 8 years demonstrated language or verbal intellectual delays, respectively, consistent with more recent outcome studies. ${ }^{11} 1633$ Wynn et al reported Bayley-III scores in the low normal range but $10 \%$ had severe delay, with risk factors for lower scores being supplemental oxygen at 28 days, a prenatal diagnosis, need for ECMO and exclusive tube feeds at discharge. ${ }^{33}$

Our findings extend the limited emerging data of heightened risk for language delay, partly due to the introduction of a separate language domain on the Bayley 
Scales. ${ }^{15}$ While these otherwise 'normal' findings may be reassuring, there are concerns that the Bayley-III underestimates impairment. Control groups of Australian children have consistently demonstrated mean scores around 107 in all domains such that the children in our cohort have probably performed worse than suggested. ${ }^{28} 29$ Somewhat reassuringly, our samples of older children also demonstrated mean intellectual scores in the normal range. Specific language assessment may be warranted, however. Moreover, the aim of this study was not to report outcome scores but rather to determine whether early neuroimaging could determine which children were most likely to have later delays. In our cohort, patient risk factors strongly associated with subsequent neurodevelopmental outcome are consistent with those previously reported as risk factors for morbidity and longer term neurodevelopmental impairment. ${ }^{1011} 13143435$

An abnormal CUS was predictive of 2-year developmental impairment but not later, presumably because ultrasound only allows for detection of significant insults, which are more likely to present early with problems, rather than the more subtle deficits which may appear later. Our rates of ultrasound anomalies were half that reported by Tracy et al, perhaps because they were undertaken so early in life, but more strongly associated with 2-year outcomes than that group's 1-year and 3-year old assessments. ${ }^{24}$ There are no other comparable data available to guide interpretation of these differences.

At 2 years, the presence of parenchymal injury on MRI was associated with lower cognitive scores. Delay in myelination and cortical folding were predictive of worse motor function. There were no MRI findings which predicted 5-year outcomes once adjustments were made for patient factors, and those maturity scores that identified the children at 8 years with more neurodevelopmental impairment were less significant, but still important, once accounting for patient factors. These are new findings with no comparable data in the existing literature. Our findings are those of low rates of significant brain injury detected and relatively normal mean neurodevelopment scores. Studies in samples with higher rates of injury and/or higher rates of neurodevelopmental impairment may be better powered to determine associations between brain injury and later outcome. The pattern of maturation delay is akin to that found in the congenital heart disease population where associations have been reported between brain maturation, particularly cortical folding, and later neurodevelopment. ${ }^{26} 36$

Strengths of this study are the high rates of routinely employed MRI in the largest reported cohort of CDH to date. While ECMO is readily available in our institution, it is rarely utilized such that these data provide a clearer picture of the impact of $\mathrm{CDH}$ itself on later outcomes.

While our overall sample size is greater than previous reports, individual age groups remain small. We will be unable to legitimately combine the many more children who will be assessed in our clinic due to the change to newer versions of the neurocognitive tests being utilized. A further limitation is the lack of control group which is challenging to ascertain when drawing from a clinical service. While preoperative ultrasound was performed routinely, the postoperative ultrasound data is likely to be biased due to the low rate of postoperative scans and the likelihood that scans were performed in the babies perceived to be at higher risk of brain injury. Data about provision of EI for this population were not available. It is possible that the lack of correlation between imaging and developmental outcomes at school age is a consequence of EI being provided to those children with the greatest developmental challenges, who were identified at 2 years. The impact of EI for this group of children requires further investigation. Assessment of children at different ages creates challenges in combining results. While the impact of $\mathrm{CDH}$ on brain development appears to diminish over time, this may be because 2-year-old follow-up overestimates impact or because of intervening influences which we have not been able to measure. However, it may simply be due to differences in the tests utilized.

Brain MRI shows promise as a tool for assessing brain maturity with a view to determining which children are at greatest risk of subsequent neurodevelopmental impairment. However, our findings suggest that surrogate markers of disease severity, such as length of time on supplemental oxygen, may also easily identify the children who will most benefit from EI such that a combination of both may focus resources on MRI scans in the highest risk children based on those clinical markers.

Key messages

What is already known about this subject?

- Congenital diaphragmatic hernia (CDH) increases the risk of both neuroimaging anomalies and neurodevelopmental impairment.

- Several perioperative clinical parameters are known to increase the risk of neurodevelopmental impairment in $\mathrm{CDH}$.

- In some other high-risk neonatal conditions, neuroimaging can identify those at highest risk of later neurodevelopmental impairment.

\section{What are the new findings?}

- Modern outcomes of CDH care, where extracorporeal life support is routinely avoided, appear to be improving.

- Preoperative cranial ultrasound has a low yield in detecting brain anomalies in $\mathrm{CDH}$.

- Cerebral MRI may detect those infants of highest risk for neurodevelopmental impairment.

How might it impact on clinical practice in the foreseeable future?

- In addition to known clinical risk factors, cerebral MRI may have a role in the routine care of high-risk $\mathrm{CDH}$ infants.

> In the absence of other abnormal morphological features, routine preoperative cranial ultrasound has limited utility in CDH screening. 
Contributors JKGC was responsible for the study protocol, data collection, analysis, the first draft of the manuscript and oversight of the research as guarantor of the integrity of the research on behalf of all authors. ACB, SM and MMM collected and analyzed data and contributed to editing of the manuscript. EAH was responsible for the study protocol and collected data. SG contributed to the study protocol, collected data and edited the manuscript. RWH was responsible for project oversight, funding access, study protocol, data analysis and editing of the manuscript.

Funding The Neonatal Neurodevelopment Follow-up Clinic is funded by The Royal Children's Hospital Foundation, Melbourne Australia.

Competing interests None declared.

Patient consent for publication Not required.

Ethics approval Human Research and Ethics Committee approval was obtained for an opt-out consent process to collate clinically acquired data for research purposes.

Provenance and peer review Not commissioned; externally peer reviewed.

Data availability statement Data may be obtained from a third party and are not publicly available.

Open access This is an open access article distributed in accordance with the Creative Commons Attribution Non Commercial (CC BY-NC 4.0) license, which permits others to distribute, remix, adapt, build upon this work non-commercially, and license their derivative works on different terms, provided the original work is properly cited, appropriate credit is given, any changes made indicated, and the use is non-commercial. See: http://creativecommons.org/licenses/by-nc/4.0/.

\section{REFERENCES}

1. McGivern MR, Best KE, Rankin J, et al. Epidemiology of congenital diaphragmatic hernia in Europe: a register-based study. Arch Dis Child Fetal Neonatal Ed 2015;100:F137-44.

2. Chiu PPL, ljsselstijn H. Morbidity and long-term follow-up in $\mathrm{CDH}$ patients. Eur J Pediatr Surg 2012;22:384-92.

3. Cortes RA, Keller RL, Townsend T, et al. Survival of severe congenital diaphragmatic hernia has morbid consequences. $J$ Pediatr Surg 2005;40:36-46. discussion 45-6.

4. Chen C, Friedman S, Butler S, et al. Approaches to neurodevelopmental assessment in congenital diaphragmatic hernia survivors. J Pediatr Surg 2007;42:1052-6. discussion 56.

5. Bagolan P, Morini F. Long-Term follow up of infants with congenital diaphragmatic hernia. Semin Pediatr Surg 2007;16:134-44.

6. Friedman S, Chen C, Chapman JS, et al. Neurodevelopmental outcomes of congenital diaphragmatic hernia survivors followed in a multidisciplinary clinic at ages 1 and 3. J Pediatr Surg 2008;43:1035-43.

7. Stolwijk LJ, Lemmers PM, Harmsen M, et al. Neurodevelopmental outcomes after neonatal surgery for major noncardiac anomalies. Pediatrics 2016;137:e20151728.

8. Van Meurs KP, Robbins ST, Reed VL, et al. Congenital diaphragmatic hernia: long-term outcome in neonates treated with extracorporeal membrane oxygenation. J Pediatr 1993;122:893-9.

9. Rocha G, Azevedo I, Pinto JC, et al. Follow-up of the survivors of congenital diaphragmatic hernia. Early Hum Dev 2012;88:255-8.

10. Danzer E, Gerdes M, Bernbaum J, et al. Neurodevelopmental outcome of infants with congenital diaphragmatic hernia prospectively enrolled in an interdisciplinary follow-up program. J Pediatr Surg 2010;45:1759-66.

11. Danzer E, Gerdes M, D'Agostino JA et al. Neurodevelopmental outcome at one year of age in congenital diaphragmatic hernia infants not treated with extracorporeal membrane oxygenation. $J$ Pediatr Surg 2015;50:898-903.

12. Safavi A, Synnes AR, O'Brien K, et al. Multi-Institutional follow-up of patients with congenital diaphragmatic hernia reveals severe disability and variations in practice. J Pediatr Surg 2012;47:836-41.

13. Danzer E, Gerdes M, D'Agostino JA, et al. Preschool neurological assessment in congenital diaphragmatic hernia survivors: outcome and perinatal factors associated with neurodevelopmental impairment. Early Hum Dev 2013;89:393-400.
14. Danzer E, Gerdes M, D'Agostino JA, et al. Longitudinal neurodevelopmental and neuromotor outcome in congenita diaphragmatic hernia patients in the first 3 years of life. $J$ Perinatol 2013;33:893-8.

15. Leeuwen L, Walker K, Halliday R, et al. Neurodevelopmental outcome in congenital diaphragmatic hernia survivors during the first three years. Early Hum Dev 2014;90:413-5.

16. Tureczek I, Caflisch J, Moehrlen U, et al. Long-Term motor and cognitive outcome in children with congenital diaphragmatic hernia. Acta Paediatr 2012;101:507-12.

17. Michel F, Baumstarck K, Gosselin A, et al. Health-Related quality of life and its determinants in children with a congenital diaphragmatic hernia. Orphanet J Rare Dis 2013;8:89.

18. Madderom MJ, Toussaint $L$, van der Cammen-van Zijp MHM, et al. Congenital diaphragmatic hernia with(out) ECMO: impaired development at 8 years. Arch Dis Child Fetal Neonatal Ed 2013;98:F316-F322.

19. Frisk V, Jakobson LS, Unger S, et al. Long-Term neurodevelopmental outcomes of congenital diaphragmatic hernia survivors not treated with extracorporeal membrane oxygenation. J Pediatr Surg 2011;46:1309-18.

20. Tracy S, Chen C. Multidisciplinary long-term follow-up of congenital diaphragmatic hernia: a growing trend. Semin Fetal Neonatal Med 2014;19:385-91.

21. Lally KP, Engle W, American Academy of Pediatrics Section on Surgery. Postdischarge follow-up of infants with congenital diaphragmatic hernia. Pediatrics 2008;121:627-32.

22. Hunt RW, Kean MJ, Stewart MJ, et al. Patterns of cerebral injury in a series of infants with congenital diaphragmatic hernia utilizing magnetic resonance imaging. J Pediatr Surg 2004;39:31-6.

23. Danzer E, Zarnow D, Gerdes M, et al. Abnormal brain development and maturation on magnetic resonance imaging in survivors of severe congenital diaphragmatic hernia. J Pediatr Surg 2012;47:453-61.

24. Tracy S, Estroff J, Valim C, et al. Abnormal neuroimaging and neurodevelopmental findings in a cohort of antenatally diagnosed congenital diaphragmatic hernia survivors. J Pediatr Surg 2010;45:958-65.

25. Brindle ME, Cook EF, Tibboel D, et al. A clinical prediction rule for the severity of congenital diaphragmatic hernias in newborns. Pediatrics 2014;134:e413-9.

26. Beca J, Gunn JK, Coleman L, et al. New white matter brain injury after infant heart surgery is associated with diagnostic group and the use of circulatory arrest. Circulation 2013;127:971-9.

27. Bayley N. Bayley scales of infant and toddler development- III. San Antonio, Texas: Psychological Corporation, 2006.

28. Chinta S, Walker K, Halliday R, et al. A comparison of the performance of healthy Australian 3-year-olds with the standardised norms of the Bayley scales of infant and toddler development (version-III). Arch Dis Child 2014;99:621-4.

29. Anderson PJ, De Luca CR, Hutchinson E, et al. Underestimation of developmental delay by the new Bayley-III scale. Arch Pediatr Adolesc Med 2010;164:352-6.

30. Acton BV, Biggs WSG, Creighton DE, et al. Overestimating neurodevelopment using the Bayley-III after early complex cardiac surgery. Pediatrics 2011;128:e794-800.

31. Wechsler D. The Wechsler Preschool and Primary Scale of Intelligence, Australian Standardisation. 3rd edn. Sydney NSW: Pearson, 2004.

32. Wechsler. Wechsler Intelligence Scale for Children. San Antonio, Texas: The Psychological Corporation, 2003.

33. Wynn J, Aspelund G, Zygmunt A, et al. Developmental outcomes of children with congenital diaphragmatic hernia: a multicenter prospective study. J Pediatr Surg 2013;48:1995-2004.

34. Putnam LR, Harting MT, Tsao K, et al. Congenital diaphragmatic hernia defect size and infant morbidity at discharge. Pediatrics 2016;138:e20162043.

35. Bevilacqua F, Morini F, Zaccara A, et al. Neurodevelopmental outcome in congenital diaphragmatic hernia survivors: role of ventilatory time. J Pediatr Surg 2015;50:394-8.

36. Licht DJ, Shera DM, Clancy RR, et al. Brain maturation is delayed in infants with complex congenital heart defects. J Thorac Cardiovasc Surg 2009;137:529-37. discussion 36-7. 